Meta

Journal des traducteurs

Translators' Journal

\title{
La nomenclature en chimie minérale
}

\section{Gilles Bélanger}

Volume 27, numéro 3, septembre 1982

URI : https://id.erudit.org/iderudit/002040ar

DOI : https://doi.org/10.7202/002040ar

Aller au sommaire du numéro

Éditeur(s)

Les Presses de l'Université de Montréal

ISSN

0026-0452 (imprimé)

1492-1421 (numérique)

Découvrir la revue

Citer cet article

Bélanger, G. (1982). La nomenclature en chimie minérale. Meta, 27(3), 295-305.

https://doi.org/10.7202/002040ar d'utilisation que vous pouvez consulter en ligne.

https://apropos.erudit.org/fr/usagers/politique-dutilisation/ 


\section{ÉTUDES \\ TERMINOLOGIQUES \\ ET LINGUISTIQUES}

\section{LA NOMENCLATURE EN CHIMIE MINÉRALE}

L'article qui suit vise à exposer le plus simplement possible les règles de dénomination des corps inorganiques en anglais et en français. Ces règles font bien sûr appel à des notions qui ne peuvent être toutes expliquées ici; on les retrouvera sans peine dans un manuel d'introduction à la chimie. Quelques notions fondamentales méritent toutefois d'être éclaircies. La plupart des noninitiés confondent en effet certains termes relativement simples, comme atome et élément, élément et composé ou composé et mélange.

\section{NOTIONS FONDAMENTALES}

L'atome est la plus petite quantité de matière susceptible de se combiner. Pour le chimiste, c'est le constituant élémentaire de la matière; il est formé d'électrons, auxquels on attribue par convention une charge négative, et d'un noyau à charge positive. Dans ce noyau (nucleus), on trouve des neutrons et des protons que l'on appelle collectivement des nucléons (nucleon) et des particules plus petites qui intéressent le physicien atomiste. Un nuclide, ou nucléide (nuclide) est un noyau de nombre atomique, ou numéro atomique (atomic number) $\mathrm{Z}$ et de nombre de masse, ou masse atomique (atomic mass) $\mathrm{A}$.

Le mot élément désigne toute configuration atomique d'un nombre $Z$ d'électrons gravitant autour d'un noyau comportant $Z$ protons; le nombre de neutrons peut varier et, par conséquent, le nombre de masse $A$. Chacun des atomes d'un même élément ayant un nombre de masse différent est un isotope. Si le nombre des éléments connus n'est que de 106, le nombre d'isotopes atteint environ 1370. Les termes atome et élément, très proches par leurs définitions, ne sont donc pas employés comme synonymes : élément se rattache à la notion d'espèce chimique, tandis qu'atome renvoit à l'idée de quantité de matière.

Corps, ou corps chimique (substance), se dit de toute substance matérielle, solide, liquide ou gazeuse, pure ou en mélange avec d'autres. Substance et corps chimique se définissent l'un par l'autre. Un corps constitué d'atomes d'un seul élément est un corps simple (simple body); ce terme s'oppose à composé ou corps composé (compound), que l'on définit comme un corps pur décomposable en de nouveaux corps purs. Ainsi, l'eau pure se décompose en oxygène pur et en hydrogène pur. Un corps pur (pure substance) est une substance ayant des propriétés physiques et chimiques bien définies. Ce terme s'oppose à mélange (mixture). Ce dernier renferme des molécules de plusieurs corps. Le pétrole brut 
est un mélange d'hydrocarbures. Les alliages sont aussi des mélanges de métaux. Les mélanges peuvent être séparés par des méthodes d'analyse immédiate (proximate analysis), telles la distillation, la cristallisation ou l'extraction au solvant; pour connaitre la composition d'un corps pur, il faut avoir recours à des méthodes d'analyse élémentaire (ultimate analysis) faisant intervenir des réactions chimiques.

On appelle molécule un agrégat d'atomes liés entre eux par des interactions physico-chimiques appelées liaisons. Ex. $\mathrm{O}_{2}, \mathrm{H}_{2} \mathrm{O}, \mathrm{CH}_{4}$. Les molécules peuvent être des corps simples ou des corps composés.

Un corps binaire (binary compound) est un corps constitué d'atomes de deux éléments différents. $\mathrm{Ex}$ : $\mathrm{H}_{2} \mathrm{O}, \mathrm{NaCl}$. De même, un corps ternaire (ternary compound) comporte des atomes de trois éléments différents et, plus généralement, un corps polyatomique (polyatomic compound) renferme des atomes de plus de deux éléments. Polyatomique se dit aussi d'agrégats de deux atomes ou plus.

Espèce chimique a deux sens en français : 1) tout élément et 2) tout corps pur. Le terme anglais species correspond au premier sens.

Les éléments se répartissent en trois grandes catégories: les métaux, ou éléments métalliques, les non-métaux, ou éléments non métalliques (nonmetal) et les gaz inertes, ou gaz rares (inert gas, rare gas). Le terme métalloïde (metalloid) a été rejeté au profit de non-métal.

\section{NOMS ET FORMULES CHIMIQUES}

La nomenclature systématique (systematic nomenclature, international system of nomenclature), c'est-à-dire le système de dénomination des atomes et de leurs combinaisons, a été élaborée et adoptée lors de congrès internationaux tenus à Genève (Système de Genève, 1892), à Liège (Union internationale de chimie, UIC, 1930) et à Amsterdam (Union internationale de chimie pure et appliquée, UICPA, 1949), puis perfectionnée lors de congrès subséquents. Notons toutefois que les efforts de systématisation remontent plus loin, à l'époque de Lavoisier et de Berzélius, ce dernier prônant un système analogue à celui de Linné pour les plantes.

Chaque élément est représenté par un symbole de une ou deux lettres, formé de l'initiale du nom de l'élément, de l'initiale et d'une autre lettre, ou d'une combinaison de lettres tirées d'un nom ancien (p. ex., $\mathrm{Hg}$, pour hydrargyre (mercure); K, pour kallium (potassium); $\mathrm{N}$, pour nitrogène (azote). Seuls les trois premiers isotopes de l'hydrogène ont reçu un nom particulier: protium, deutérium (D) et tritium (T). Les autres sont désignés par des nombres : carbone14, par exemple.

La nomenclature comporte encore de nombreux noms triviaux ou noms vulgaires (trivial name, common name), dérivant arbitrairement de la langue commune et ne dénotant aucunement la structure chimique du corps en question : eau, chaux, soude, fer, brome, etc.

D'autres ont une origine triviale, mais leur terminaison possède une signification systématique. Ainsi, près des deux tiers des éléments se terminent en ium. De même pour la terminaison ol indiquant un alcool. Ce sont des noms semi- 
triviaux (semitrivial name). Enfin, la plupart des corps chimiques possèdent un nom systématique (systematic name) décrivant la structure du corps dénommé et pouvant être dérivé suivant des règles précises.

Un nom fondamental (fundamental name, parent name) est un nom systématique (qui peut aussi être d'origine triviale ou semi-triviale) désignant un corps considéré comme la structure initiale dont dérivent d'autres corps, par addition, soustraction, substitution d'atomes ou de groupes d'atomes. Par exemple, méthane, éthane, propane et butane sont des noms semi-triviaux. Pentane (penta + ane) et hexane (hexa + ane) sont des noms systématiques. Tous les six sont aussi des noms fondamentaux, qui peuvent être modifiés par adjonction d'affixes indiquant une modification de la molécule : par exemple, cyclo hexane, chloro-éthane, pentanone.

La formule chimique (chemical formula) est une écriture symbolique représentant la molécule. Elle peut prendre plusieurs formes. La formule brute, ou formule générale (empirical formula, general formula) indique seulement le nombre relatif d'atomes différents dans la molécule. La formule moléculaire (molecular formula) dénote le nombre réel d'atomes de chaque élément, mais pas nécessairement la structure de la molécule. Par exemple, la formule brute $\mathrm{NO}_{2}$ correspond à deux corps de formules moléculaires $\mathrm{NO}_{2}$ et $\mathrm{N}_{2} \mathrm{O}_{4} . \mathrm{Cx}\left(\mathrm{H}_{2} \mathrm{O}\right)_{y}$ est la formule générale des glucides, tandis que $\mathrm{C}_{6} \mathrm{H}_{12} \mathrm{O}_{6}$ est la formule moléculaire du glucose et du fructose.

Pour représenter une différence de structure (par exemple, entre le glucose et le fructose), il faut écrire une formule développée, aussi appelée formule structurale ou représentation plane (structural formula, graphic formula), indiquant dans un plan l'ordre des atomes et symbolisant les liaisons par des traits. Enfin, pour les besoins de l'analyse conformationnelle et de l'étude des stéréoisomères, il peut être nécessaire de représenter la molécule suivant les trois dimensions : c'est la formule ou représentation stéréochimique (space representation).

\section{LA NOMENCLATURE EN CHIMIE MINÉRALE}

La nomenclature systématique attribue à chaque corps un nom le plus descriptif possible et fait un usage systématique d'affixes de formation savante. À côté de cette nomenclature subsiste l'ancienne, davantage fondée sur des noms triviaux. Dans les paragraphes qui suivent, nous mentionnerons les deux systèmes.

1. Le graphique ci-joint résume les principales combinaisons. Les métaux sont des corps électropositifs et forment des cations. Les non-métaux, généralement électronégatifs, se retrouvent dans l'anion. L'hydrogène et l'oxygène peuvent réagir avec presque tous les éléments, tandis que les gaz rares ne réagissent que très exceptionnellement. Les métaux ne réagissent pas entre eux, mais forment des alliages (alloy). Les non-métaux réagissent entre eux et avec les métaux.

2. L'écriture symbolique place le corps le plus électropositif (ou considéré comme tel) en première position, et la nomenclature anglaise respecte cet 


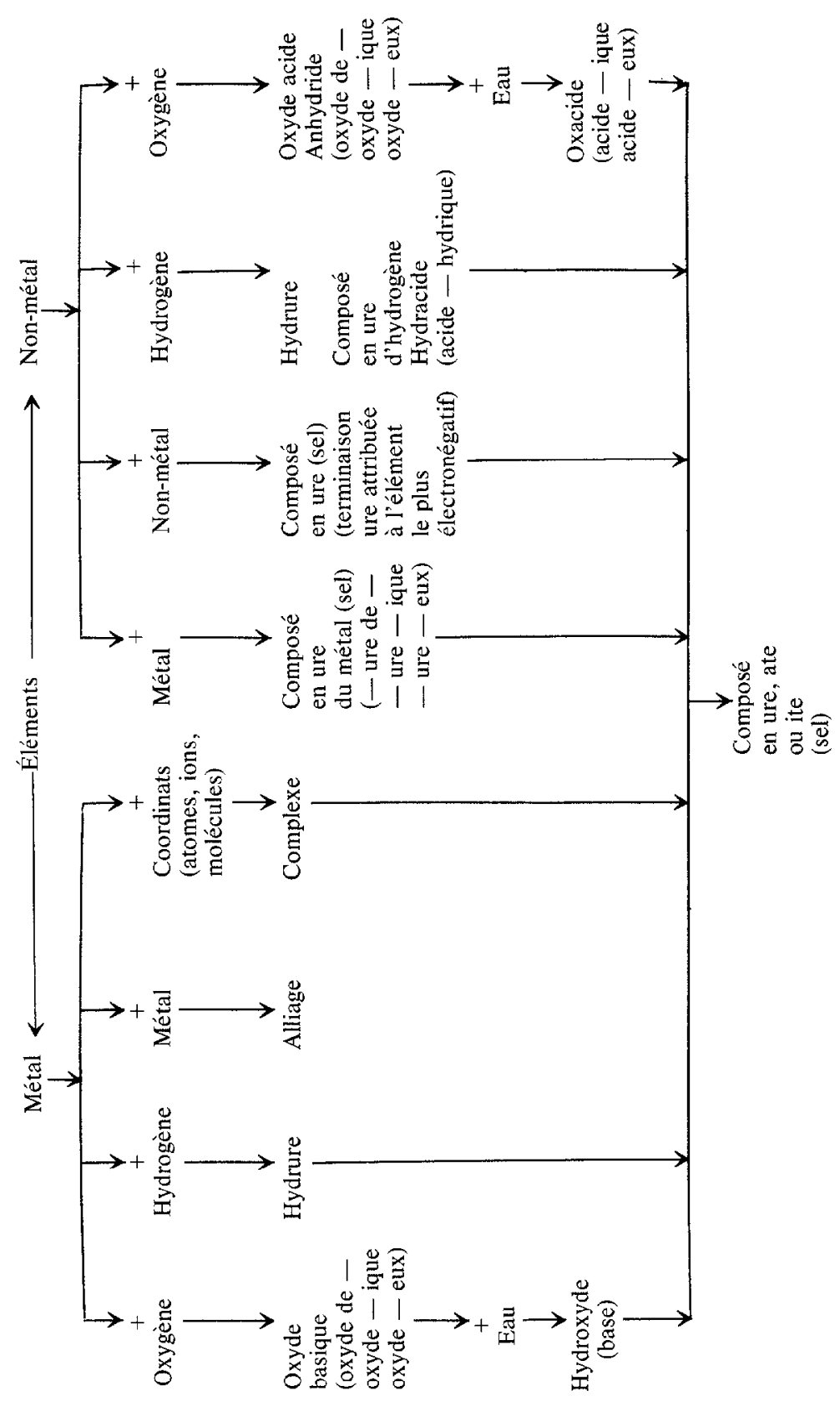


ordre. Dans la nomenclature française, cet ordre est inversé, suivant le schéma habituel, déterminé-déterminant. Le nom du déterminant n'est pas modifié.

Sodium chloride $\quad \mathrm{Na}^{+} \mathrm{Cl}^{-} \quad$ Chlorure de sodium

\section{COMPOSÉS BINAIRES}

3. Pour nommer un composé binaire en anglais, on ajoute au déterminé la terminaison ide, sauf dans certains composés ayant conservé un nom trivial (p. ex., $\mathrm{NH}_{3}$, ammonia; $\mathrm{H}_{2} \mathrm{O}$, water, etc.) et pour les composés binaires du silicium et de l'hydrogène (silicon hydrides ou silanes) et ceux du bore et de l'hydrogène (boron hydrides ou boranes).

4. En français, la terminaison correspondante est ure, mais en plus des exceptions déjà mentionnées au paragraphe précédent, il faut ajouter les composés binaires de l'oxygène, qui sont des oxydes : oxydes basiques en combinaison avec les métaux, et oxydes acides ou anhydrides avec les non-métaux et certains métaux à certains degrés ou nombres d'oxydation (oxidation state, oxidation number) (nombre d'électrons reçus ou donnés).

5. Les composés de l'hydrogène et des métaux sont des hydrures (hydride) car l'hydrogène y joue le rôle d'anion $\left(\mathrm{H}^{-}\right)$.

Potassium hydride $\mathrm{KH}$ hydrure de potassium

Ceux de l'hydrogène et des non-métaux forment plutôt leurs noms en ajoutant au radical du non-métal la terminaison ure (p. ex. phosphure d'hydrogène, $\mathrm{H}_{3} \mathrm{P}$ ), sauf les exceptions nommées en 3 (eau, ammoniac, silanes, boranes, etc.)

6. Les halogénures d'hydrogène (hydrogen halides) sont des hydracides et sont appelés de préférence acide - hydrique. En anglais, ils sont nommés suivant les règles de la nomenclature systématique ( $\mathrm{p}$. ex. hydrogen chloride), la forme hydro - ic acid étant réservée en général à la solution dans l'eau.

7. Dans leurs combinaisons avec l'oxygène, bon nombre d'éléments métalliques et non métalliques peuvent prendre plusieurs degrés d'oxydation, donnant autant d'oxydes aux rapports stoéchiométriques différents. L'ancienne nomenclature utilise les suffixes eux (ous) et ique (ic) respectivement pour le composé le moins et le plus oxydé. Si un élément peut former plus de deux oxydes, on utilise de plus les préfixes hypo (hypo) et per (per). En anglais, on emploie de préférence des préfixes numériques. Certains composés de l'oxygène et des non-métaux sont aussi appelés anhydrides (anhydride). (Voir par. 13.)

$\begin{array}{rll}\text { Nitrous oxide or dinitrogen oxide } & \mathrm{N}_{2} \mathrm{O} & \text { oxyde nitreux ou protoxyde d'azote } \\ \text { Nitric oxide or nitrogen oxide } & \mathrm{NO} & \text { oxyde nitrique } \\ \text { Dinitrogen trioxide } & \mathrm{N}_{2} \mathrm{O}_{3} & \text { anhydride nitreux } \\ \text { Dinitrogen pentaoxide } & \mathrm{N}_{2} \mathrm{O}_{5} & \text { anhydride nitrique }\end{array}$

Il existe des oxydes multiples (multiple oxide, mixed oxide) de métaux. C'est improprement qu'on leur donne des noms du genre «titanate de fer». (Voir par. 12.)

Iron titanium trioxide $\quad \mathrm{FeTiO}_{3} \quad$ trioxyde de fer et de titane

8. La terminaison ide anglaise n'est pas réservée uniquement aux composés binaires de la chimie minérale. Il existe par exemple des composés azotés appelés, tant en anglais qu'en français, des amides et des imides. 


\section{COMPOSÉS POLYATOMIQUES}

9. Les composés polyatomiques sont formés d'atomes de plus de deux éléments différents. Différents types de composés peuvent entrer dans cette catégorie. Les principaux sont des hydroxydes, des oxacides (oxoacid) et des sels.

10. Les hydroxydes sont des composés d'un métal et du groupement hydroxyle $\left(\mathrm{OH}^{-}\right)$.

$$
\text { Sodium hydroxide } \quad \mathrm{NaOH} \quad \text { hydroxyde de sodium }
$$

Il existe aussi des hydroxydes multiples.

11. Les oxacides sont des composés dont l'anion renferme un non-métal associé à l'oxygène. Le nom de ces composés est formé en modifiant le nom de l'atome central en ique (ic) si cet atome ne possède qu'un degré d'oxydation. Si l'atome central possède plus d'un degré d'oxydation, on emploie les suffixes ique (ic) et eux (ous) et les préfixes hypo et per. (Voir par. 13.)

$\begin{array}{rll}\text { Sulfuric acid } & \mathrm{H}_{2} \mathrm{SO}_{4} & \text { acide sulfurique } \\ \text { Sulfurous acid } & \mathrm{H}_{2} \mathrm{SO}_{3} & \text { acide sulfureux } \\ \text { Perchloric acid } & \mathrm{HClO}_{4} & \text { acide perchlorique } \\ \text { Chloric acid } & \mathrm{HClO}_{3} & \text { acide chlorique } \\ \text { Chlorous acid } & \mathrm{HClO}_{2} & \text { acide chloreux } \\ \text { Hypochlorous acid } & \mathrm{HClO} & \text { acide hypochloreux }\end{array}$

Les préfixes ortho et méta sont aussi employés pour indiquer la «teneur en eau ».

$\begin{array}{cll}\text { Orthophosphoric acid } & \mathrm{H}_{3} \mathrm{PO}_{4} & \text { acide orthophosphorique } \\ \text { Trimetaphosphoric acid } & \left(\mathrm{HPO}_{3}\right)_{3} & \text { acide trimétaphosphorique }\end{array}$

Le préfixe pyro a aussi été employé pour désigner un acide formé de deux molécules d'un acide ortho, moins une molécule d'eau.

Pyrosulfuric or
disulfuric acid $\mathrm{H}_{2} \mathrm{~S}_{2} \mathrm{O}_{7} \quad$ acide pyrosulfurique.

Le préfixe peroxo (peroxo, peroxy) indique le remplacement d'un atome d'oxygène par un groupement peroxo (-O-O-).

$\begin{array}{rll}\begin{array}{r}\text { Peroxonitric acid } \\ \text { Peroxy(mono)sulfuric acid }\end{array} & \begin{array}{l}\mathrm{HNO}_{4} \\ \mathrm{H}_{2} \mathrm{SO}_{5}\end{array} & \begin{array}{l}\text { acide peroxonitrique }\left(\mathrm{NO}_{2}-\mathrm{O}-\mathrm{OH} \text { ) }\right. \\ \text { acide peroxo(mono)sulfurique } \\ \text { ( } \mathrm{HSO}-\mathrm{O}-\mathrm{OH})\end{array} \\ \text { Peroxydisulfuric acid } & \mathrm{H}_{2} \mathrm{~S}_{2} \mathrm{O}_{8} & \begin{array}{l}\text { acide peroxodisulfurique } \\ \left(\mathrm{HSO}_{3}-\mathrm{O}-\mathrm{O}-\mathrm{SO}_{3} \mathrm{H}\right)\end{array}\end{array}$

Le remplacement de l'oxygène par le soufre s'indique par le préfixe thio (thio).

$\begin{array}{cll}\text { (Mono)thiophosphoric acid } & \mathrm{H}_{3} \mathrm{PO}_{3} \mathrm{~S} & \text { acide (mono) thiophosphorique } \\ \text { Dithiophosphoric acid } & \mathrm{H}_{3} \mathrm{PO}_{2} \mathrm{~S}_{2} & \text { acide dithiophosphorique }\end{array}$

Les préfixes séléno et telluro s'emploient de la même façon.

12. Les sels résultent de diverses combinaisons, mais pour les besoins de la nomenclature, on considère qu'ils dérivent soit d'un hydracide, soit d'un oxacide.

Le nom des sels dérivant des hydracides (ne contenant pas d'oxygène) est formé du nom de l'anion et de la terminaison ure (ide).
Potassium chloride
$\mathrm{KCl}$
chlorure de potassium
Potassium cyanide
$\mathrm{KCN}$
cyanure de potassium

Dans le cas des sels dérivant des oxacides (qui contiennent de l'oxygène), deux cas peuvent se produire: 1) si le nom de l'oxacide se termine en ique (ic), le nom du sel prend la terminaison ate (ate);2) si le nom de l'oxacide se termine en eux (ous), le nom du sel prend la terminaison ite (ite). (Voir par. 13.) 


\begin{tabular}{|c|c|c|}
\hline Sodium carbonate & $\mathrm{Na}_{2} \mathrm{CO}_{3}$ & carbonate de sodium \\
\hline Sodium nitrite & $\mathrm{NaNO}_{2}$ & $\begin{array}{l}\text { nitrite de sodium } \\
\text { (dérive de l'acide nitreux) }\end{array}$ \\
\hline Sodiu & $\mathrm{NaNO}_{3}$ & $\begin{array}{l}\text { nitrate de sodium } \\
\text { (dérive de l'acide nitrique) }\end{array}$ \\
\hline
\end{tabular}

Les sels acides (acid salt) renferment de l'hydrogène acide remplaçable, dont la présence est indiquée, en français, 1) en ajoutant le mot acide après le nom de l'anion; 2) en préfixant le mot hydrogéno (ou dihydrogéno) au nom de l'anion; ou 3) en ajoutant un préfixe numérique au nom du cation. En anglais, cet hydrogène acide est indiqué par le mot hydrogen placé devant le nom de l'anion.

Sodium hydrogen carbonate

Sodium dihydrogen phosphate

Sodium (mono)hydrogen phosphate
$\mathrm{NaHCO}_{3}$ carbonate acide de sodium ou bicarbonate de sodium

$\mathrm{NaH}_{2} \mathrm{PO}_{4}$ dihydrogénophosphate de sodium ou phosphate monosodique

$\mathrm{Na}_{2} \mathrm{HPO}_{4}$ hydrogénophosphate de sodium ou phosphate disodique

Les sels doubles et triples sont des sels dans lesquels deux ou trois cations d'espèces chimiques différentes sont réunis au même anion. On peut alors, en français, ajouter l'adjectif double ou triple après le nom de l'anion.

Potassium sodium carbonate $\quad \mathrm{KNaCO}_{3} \quad$ carbonate double de potassium et de sodium

Les sels basiques (basic salt) sont des sels doubles renfermant des anions $\mathrm{O}^{2-}$ et $\mathrm{OH}^{-}$, dont la présence est indiquée en français en ajoutant au nom de l'anion les préfixes oxy ou hydroxy respectivement. En anglais, le nom de ces ions n'est pas modifié.

Magnesium hydroxide chloride $\quad \mathrm{Mg}(\mathrm{OH}) \mathrm{Cl} \quad$ hydroxychlorure de magnésium Phosphorus oxide trichloride $\mathrm{POCL}_{3}$ oxychlorure de phosphore

\section{NOMENCLATURE SYSTÉMATIQUE}

13. L'ancienne nomenclature (a.n.) utilise des préfixes et des suffixes (hypo, per, ique, eux) pour indiquer le degré d'oxydation et, par conséquent, les rapports stoéchiométriques. L'inconvénient de ce mode de notation est qu'aucun de ces affixes n'est rattaché à un degré d'oxydation particulier; ils n'ont de signification que par rapport à d'autres composés d'une série donnée.

Dans la nomenclature systématique (n.s.), les rapports stoéchiométriques peuvent être indiqués par des préfixes numériques grecs ou latins : mono, bi ou di, tri, tétra, penta, hexa, etc.

\begin{tabular}{|c|c|c|}
\hline Nitrous oxide (a.n.) & $\mathrm{N}_{2} \mathrm{O}$ & oxyde nitreux \\
\hline Dinitrogen oxide (n.s.) & & protoxyde d'azote \\
\hline Nitric oxide (a.n.) & NO & oxyde nitrique \\
\hline nitrogen monoxide (n.s.) & & monoxyde d'azote \\
\hline Nitrogen dioxide & $\mathrm{NO}_{2}$ & bioxyde ou dioxyde d'azote \\
\hline Dinitrogen trioxide & $\mathrm{N}_{2} \mathrm{O}_{3}$ & $\begin{array}{l}\text { anhydride nitreux (a.n.) } \\
\text { sesquioxyde d'azote (n.s.) }\end{array}$ \\
\hline Disulphur heptaoxide & $\mathrm{S}_{2} \mathrm{O}_{7}$ & hémiheptaoxyde de soufre \\
\hline
\end{tabular}

Ces préfixes peuvent toutefois être omis lorsqu'il n'y a pas de risque de $\mathrm{S}_{2} \mathrm{O}_{7}$, par exemple, se nomme en anglais disulphur heptaoxide : déterminé et déterminant sont précédés d'un préfixe indiquant la quantité de chaque élément. En français, heptaoxyde de disoufre n'est pas naturel, de sorte que l'on a recours à un préfixe plus complexe, exprimant un rapport: hémihepta (7/2). De même, sesqui exprime un rapport de $3 / 2$. 
Ces préfixes peuvent toutefois être omis lorsqu'il n'y a pas de risque de confusion.

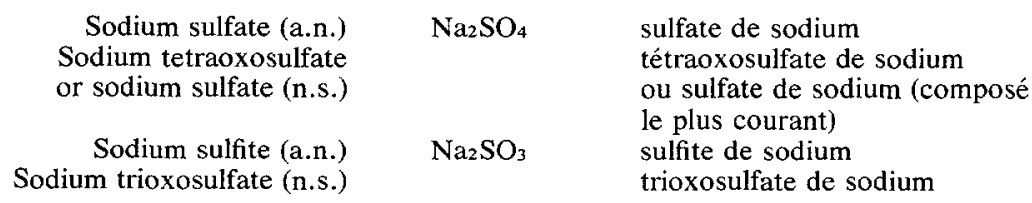

14. On peut aussi indiquer les rapports stoéchiométriques à l'aide de la notation de Stock, c'est-à-dire par des chiffres romains entre parenthèses, représentant le nombre d'oxydation de l'élément qui précèđe.

Ferrous chloride (a.n.) $\quad \mathrm{FeCl}_{2} \quad$ chlorure ferreux Iron (II) chloride chlorure de fer (II) or ferrum (II) chloride (n.s.)

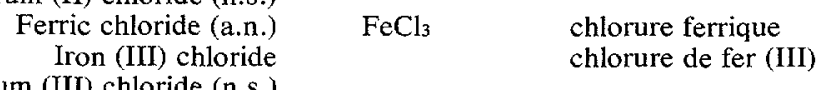
$\begin{array}{rll}\text { Ferric chloride (a.n.) } & \mathrm{FeCl}_{3} & \text { chlorure ferrique } \\ \text { Iron (III) chloride } & & \text { chlorure de fer (III) } \\ \text { or ferrum (III) chloride (n.s.) } & & \text { aciole }\end{array}$

Sulfurous acid (a.n.) $\quad \mathrm{H}_{2} \mathrm{SO}_{3} \quad$ acide sulfureux

Trioxosulfuric (IV) acid (n.s.) $\quad$ acide trioxosulfurique (IV) Sulfuric acid (a.n.) $\quad \mathrm{H}_{2} \mathrm{SO}_{4} \quad$ acide sulfurique

Tetraoxosulfuric (VI) acid (n.s.) $\quad$ acide tétraoxosulfurique (VI)

Potassium hexacyanoferrate (II) $\quad \mathrm{K}_{4}\left[\mathrm{Fe}(\mathrm{CN})_{6}\right] \quad$ hexacyanoferrate (II) de potassium

Cette notation fait disparaître, en plus des préfixes hypo et per, la distinction entre les terminaisons ique et eux d'une part, et ate et ite d'autre part. Seules sont conservées les terminaisons ique et ate.

IONS

15. Le nom des cations monoatomiques n'est pas modifié, sauf pour indiquer le degré d'oxydation.

$\begin{array}{rll}\begin{array}{r}\text { Sodium ion } \\ \text { Cuprous ion }\end{array} & \mathrm{Na}^{+} & \begin{array}{l}\text { ion sodium } \\ \text { ion culivrenx } \\ \text { ou ion cuivre (I) }\end{array} \\ \text { or copper (I) ion } & & \begin{array}{l}\text { on } \\ \text { ing }\end{array}\end{array}$

Certains cations polyatomiques correspondent à des radicaux (voir par. 15) auxquels on a donné des noms particuliers.

Nitrosyl ion (or cation or radical) $\quad \mathrm{NO}^{+} \quad$ ion (ou cation ou radical) nitrosyle Nitryl ion (or cation or radical) $\quad \mathrm{NO}_{2}{ }^{+} \quad$ ion (ou cation ou radical) nitryle* * À ne pas confondre avec nitrile, en chimie organique $(-\mathrm{C} \equiv N)$.

D'autres cations polyatomiques proviennent de l'addition à des cations monoatomiques d'autres ions, molécules ou atomes neutres: ce sont des complexes (voir par. 17) et leur nom n'est pas modifié.
Hexaamminecobalt ion
$\mathrm{Co}\left(\mathrm{NH}_{3}\right)_{6}{ }^{3+}$ ion hexaammine-cobalt
Chloropentaamminecobalt ion
$\mathrm{CoCl}\left(\mathrm{NH}_{3}\right) 5^{2+}$ ion chloropentaammine-cobalt

Les noms des cations provenant de l'addition de protons à des anions monoatomiques sont formés par l'addition de la terminaison onium à la racine du nom de l'anion.

$$
\begin{array}{rll}
\begin{array}{r}
\text { Phosphonium ion } \\
\text { Oxonium ion }
\end{array} & \mathrm{PH}_{4}{ }^{+} & \begin{array}{l}
\text { ion phosphonium } \\
\text { ion oxonium }
\end{array} \\
\begin{aligned}
\text { Exception: } \\
\text { Ammonium ion }
\end{aligned} & \mathrm{NH}_{4} \mathrm{O}^{+} & \begin{array}{l}
\text { ion ammonium (et non azotonium } \\
\text { ou nitronium) }
\end{array}
\end{array}
$$

Les ions ammonium substitués dérivés de bases azotées dont le nom se termine en amine forment leur nom en ammonium. 
Lorsque la base azotée ne se termine pas en amine, le nom du cation est formé en ajoutant la terminaison ium au nom de la base.
Hydrazinium $(1+)$ ion
$\mathrm{N}_{2} \mathrm{H}_{5}+$
ion hydrazinium $(1+)$
Hydrazinium $(2+)$ ion
$\mathrm{N}_{2} \mathrm{H}_{6}{ }^{2+}$
ion hydrazinium $(2+)$

Lorsque le cation est formé par l'addition de protons à un acide, le mot acidium est ajouté au nom de l'anion correspondant, et non à celui de l'acide lui-même.

$\begin{array}{lll}\text { Nitrate acidium ion } & \mathrm{H}_{2} \mathrm{NO}_{3}{ }^{+} & \text {ion nitrate-acidium } \\ \text { Nitrite acidium ion } & \mathrm{H}_{2} \mathrm{NO}_{2}{ }^{+} & \text {ion nitrite-acidium }\end{array}$

Le nom des anions monoatomiques est formé en ajoutant la terminaison ure (ide) au nom de l'élément.

$$
\begin{array}{cll}
\text { Bromide ion } & \mathrm{Br}^{-} & \text {ion bromure } \\
\text { Sulfide ion } & \mathrm{S}^{2-} & \text { ion sulfure }
\end{array}
$$

Certains anions polyatomiques prennent aussi la terminaison ure (ide).

$\begin{array}{rll}\text { Disulfide ion } & \mathrm{S}_{2}{ }^{2-} & \text { ion disulfure } \\ \text { Triiodide ion } & \mathrm{I}_{3^{-}} & \text {ion triiodure } \\ \text { Cyanide ion } & \mathrm{CN}^{-} & \text {ion cyanure } \\ \text { Amide ion } & \mathrm{NH}^{2-} & \text { ion amidure } \\ \text { Imide ion } & \mathrm{NH}^{2-} & \text { ion imidure } \\ \text { Hydrogen sulfide ion } & \mathrm{HS}^{-} & \text {ion hydrogénosulfure } \\ \text { Nitride ion } & \mathrm{N}^{3-} & \text { ion nitrure }\end{array}$

D'autres anions polyatomiques prennent en français une terminaison autre que ure.

$\begin{array}{rll}\text { Azide ion } & \mathrm{N}_{3-}^{-} & \text {ion azide } \\ \text { Hydroxide ion } & \mathrm{OH}^{-} & \text {ion hydroxyle ou oxhydrile } \\ \text { Ozonide ion } & \mathrm{O}_{3-}^{-} & \text {ion ozonide }\end{array}$

Les noms des autres anions polyatomiques sont formés en ajoutant au nom de l'atome central la terminaison ate (ate).
Tetrachloroaluminate ion
$\mathrm{AlCl}_{4}^{-}$
ion tétrachloroaluminate

Dans l'ancienne nomenclature, la terminaison ite (ite) et les préfixes hypo et per indiquent le degré d'oxydation. Toutefois, la notation de Stock peut aussi être employée.

$\begin{array}{rll}\begin{array}{r}\text { Hypochlorite ion (a.n.) } \\ \text { or chlorate (I) ion (n.s.) } \\ \text { Chlorite ion (a.n.) }\end{array} & \mathrm{ClO}^{-} & \begin{array}{l}\text { ion hypochlorite } \\ \text { ou ion chlorate (I) } \\ \text { ion chlorite } \\ \text { ou ion chlorate (III) }\end{array} \\ \begin{array}{cl}\mathrm{ClO}^{-} & \end{array} & & \end{array}$

\section{RADICAUX}

16. Les radicaux (radical) sont des groupements d'atomes susceptibles de se séparer en bloc d'une molécule pour passer dans une autre molécule de structure différente. Les radicaux momentanément non recombinés sont appelés radicaux libres (free radical). Un même radical peut se rencontrer dans un grand nombre de composés, et peut parfois avoir des fonctions différentes dans des molécules de structure différente.

Bon nombre de radicaux renfermant de l'oxygène ou un autre chalcogène ( $\mathrm{S}, \mathrm{Se}, \mathrm{Te}$ ) ont reçu des noms se terminant en yle ou osyle. On peut considérer que la plupart de ces noms (sauf hydroxyle et thionyle) proviennent du nom de l'acide correspondant, dont la terminaison en ique ou en eux a été remplacée par les terminaisons yle et osyle respectivement. 


$\begin{array}{rll}\text { Hydroxyl } & -\mathrm{OH} & \text { hydroxyle } \\ \text { Carbonyl } & =\mathrm{CO} & \text { carbonyle } \\ \text { Nitryl } & -\mathrm{NO}_{2} & \text { nitryle } \\ \text { Nitrosyl } & -\mathrm{NO} & \text { nitrosyle } \\ \text { Thionyl or sulfinyl } & =\mathrm{SO} & \text { thionyle } \\ \text { Sulfuryl or sulfonyl } & =\mathrm{SO}_{2} & \text { sulfuryle } \\ \text { Uranyl (VI) } & =\mathrm{UO}_{2} & \text { uranyle (VI) } \\ \text { Uranyl (V) } & -\mathrm{UO}_{2} & \text { uranyle (V) } \\ \text { Phosphoryl } & =\mathrm{PO} & \text { phosphoryle } \\ \text { Thiophosphoryl } & \equiv \mathrm{PS} & \text { thiophosphoryle } \\ \text { Vanadyl (V) } & \equiv \mathrm{VO} & \text { vanadyle (V) } \\ \text { Vanadyl (IV) } & =\mathrm{VO} & \text { vanadyle (IV) } \\ \text { Vanadyl (III) } & -\mathrm{VO} & \text { vanadyle (III) }\end{array}$

Le même radical peut avoir des noms différents en chimie minérale et en chimie organique.

\title{
COMPLEXES
}

17. Les complexes ou composés de coordination (complex, coordination compound) sont des édifices formés de un ou plusieurs atomes centraux liés par coordinence à plusieurs groupements périphériques (ions ou molécules) appelés coordinats ou ligands (ligand).

Dans l'écriture symbolique, le complexe est mis entre crochets et l'atome central est placé en premier. Dans le nom du complexe, les coordinats sont énoncés en tête; on commence par ceux qui sont négatifs et on les affecte de la terminaison o. Viennent ensuite les coordinats neutres et positifs, sans terminaison, et finalement le nom de l'atome central, suivi de la terminaison ate (ate) s'il s'agit d'un complexe anionique (formé autour d'un anion), sans terminaison s'il s'agit d'un complexe cationique. Le degré d'oxydation est indiqué en chiffres romains entre parenthèses.

Chloropentaamminecobalt (III) ion $\quad\left[\mathrm{CoCl}\left(\mathrm{NH}_{3}\right)_{5}\right]^{2+}$ ion chloropentaammine-cobalt (III) Potassium oxotetrafluorochromate (V)

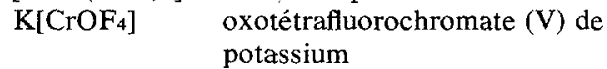
potassium

\author{
Hexaamminecobalt (III) \\ Chloride sulfate \\ Tetraamminecopper (II) ion
}

$\left[\mathrm{Co}\left(\mathrm{NH}_{3}\right)_{6}\right]_{\mathrm{ClSO}}{ }_{4}$ chlorosulfate d'hexaammine-cobalt (III)

$\left[\mathrm{Cu}\left(\mathrm{NH}_{3}\right)\right]_{4^{2+}}$ ion tétraammine-cuivre (II) HYDRATES

18. L'eau peut se fixer à certains corps pour donner des hydrates (hydrate). Il est d'usage d'indiquer, dans la formule chimique, le nombre de molécules d'eau après un point (auteurs anglophones) ou une virgule (auteurs francophones).

Copper sulfate pentahydrate

$\mathrm{CuSO}_{4} .5 \mathrm{H}_{2} \mathrm{O}$

$$
\text { ou }
$$

$\mathrm{CuSO}_{4}, 5 \mathrm{H}_{2} \mathrm{O}$ sulfate de cuivre pentahydraté

Quand le nombre de molécules d'eau est très grand, on l'indique par un nombre arabe suivi du mot eau (water). Dans les complexes, le coordinat eau s'indique par le nom latin aquo (aquo).

L'adjectif anhydre (anhydrous) indique l'absence d'eau.

Anhydrous copper sulfate $\quad \mathrm{CuSO}_{4} \quad$ sulfate de cuivre anhydre

Gilles Bélanger 


\section{BIBLIOGRAPHIE}

International Union of Pure and Applied Chemistry (1970): Nomenclature of Inorganic Chemistry, $2^{\text {nd }}$ ed., Buttersworth.

IUPAC (1981): Nomenclature of Inorganic Chemistry, American Version, in Handbook of Chemistry and Physics, $61^{\text {th }}$ ed., CRC Press.

Union internationale de chimie pure et appliquée (1976): Nomenclature de chimie minérale, Feuillet de documentation NF T/01-003, 1965, dans Recueil de normes françaises - Chimie, analyse, normes fondamentales, AFNOR.

Dictionnaire encyclopédique Quillet (1975): divers articles.

Encyclopédie Universalis (1968) : à l'article «Chimie, nomenclature».

Encyclopédie internationale des sciences et des techniques (1970): Larousse, à l'article «Chimie».

BERNARD, M. et F. BUSNOT (1978) : Aide-mémoire Dunod-Chimie générale et minérale, rale, tome $l$. 